\title{
¿Estamos valorizando correctamente los recursos naturales? \\ El caso del cobre chileno en perspectiva histórica $(1800-2021)$.
}

\section{Are we measuring natural resources correctly? The case of Chilean copper in historical perspective (1800 - 2021).}

\author{
Cristián Ducoing * \\ DEPARTAMENTO DE HISTORIA ECONÓMICA, UNIVERSIDAD DE LUND, SUECIA
}

\section{RESUMEN}

Durante el año 2021 se ha producido un aumento significativo del precio del cobre en los mercados internacionales. Este fenómeno genera una serie de preguntas, como si este valor será sostenido en el tiempo y cómo se compara el precio actual con los valores históricos. En la presenta nota de investigación, se pone en duda la relevancia del precio actual como una excepcionalidad y se proponen varias líneas de investigación en las cuales la historia económica podría aportar para el debate de los precios y valorización de los recursos naturales más allá de un simple precio de transacción.

Palabras clave: cobre, Chile, recursos naturales, sostenibilidad

\begin{abstract}
During the 2021, there has been a noticeable increase in copper prices, with the consequent analysis of whether this value will be sustained over time and how the current price compares with their historical values. In the present research note, the relevance of the current price as exceptional fact is under question. Moreover, several lines of research are proposed in which economic history could contribute to the debate on prices and natural resources valuation beyond market prices. Keywords: Copper, Chile, natural resources, sustainability
\end{abstract}

*cristian.ducoing@ekh.lu.se 
1 pasado 7 de mayo algunas notas de prensa calificaron que el cobre alcanzaba su mayor precio "histórico". El precio en cuestión fue de 4,7 dólares la libra de cobre, que se traduce en términos agregados sobre los 10 mil dólares la tonelada del mineral ${ }^{1}$. La inmediata pregunta que surge es si la afirmación el mayor precio histórico es correcta desde la perspectiva de la historia económica. Más allá de la valoración monetaria del mencionado mineral, los actuales escenarios catastróficos que nos presenta el cambio climático, obligan a preguntarse que valoración estamos haciendo de los recursos naturales y si este altísimo precio responde realmente a las externalidades que produce la explotación de los productos del subsuelo, y a la vez, si los beneficios de la explotación de los recursos no renovables están siendo re-invertidos en futuro bienestar para la población.

La función del cobre en mercado mundial ha ido evolucionando a lo largo de los últimos doscientos años. Por suerte para los objetos de la investigación científica, para los historiadores económicos y la sociedad en general, existe una importante evidencia cuantitativa con respecto al precio del producto durante este periodo. (Wright, 2015; Ducoing et al., 2018), por lo que estamos en condiciones de poner en duda, o al menos relativizar, la afirmación de los periodistas del reciente precio "histórico". A la vez, los precios de los minerales estratégicos, en cuanto a la nueva (potencial) economía verde, generan una mayor atención hacia procesos anteriores de ciclos de altos valores para las commodities.

¿Qué es el precio del cobre? Esta pregunta no es baladí si queremos valorizar el precio de un commodity en el largo plazo. Actualmente, se utiliza como referencia el precio transado en la bolsa de metales de Londres ${ }^{2}$. No obstante, como bien lo han recalcado Badia et.al (2021), para precios en el largo plazo, este valor de referencia puede esconder una serie de imprecisiones, especialmente, los distintos tipos de productos exportados por los países y los precios de exportación por grandes cantidades. Otro asunto no menor dice relación con el valor del producto con respecto a otros bienes de exportación e importación, en los denominados 
términos de intercambio. El precio del cobre puede haber subido en la actualidad o en otros momentos de la historia, pero eso no significa que las ganancias de los países productores hayan sido netas si se vieron compensadas por alzas en productos de importación. Por último, en una discusión teórica más compleja, el precio del cobre es referencial y no se condice necesariamente con el valor presente neto del producto. Si las rentas extraídas del mineral no alcanzan a compensar la depreciación del producto para las futuras generaciones, la extracción del recurso no renovable no estaría siendo beneficiosa en un marco temporal más extenso. Esto punto ya es sustancial en estudios históricos de sostenibilidad, como los resultados aportados por (Blum, Ducoing and McLaughlin, 2017), donde de los países latinoamericanos analizados, Chile resultó ser el peor en términos de ahorros genuinos ${ }^{3}$, porque los beneficios de la gran minería del cobre no se veían repercutidos en un mayor bienestar futuro de la población, determinado por una enorme explotación de los recursos naturales y a la vez, una baja inversión en capital físico y humano. La primera condición de la sostenibilidad débil, un reemplazo del capital consumido, no se estuvo cumpliendo en buena parte del siglo XX. Esta situación pone en perspectiva el debate sobre la valorización de los recursos no renovables, porque la base de la solidaridad intergeneracional propuesta por (Hartwick, 1977) no se estaría concretando en el caso de Chile y de muchos otros países dependientes de las materias primas.

Dados estos antecedentes, el rol del precio de los commodities, y en este particular caso, el del cobre, requiere una particular atención y re-estimación. Más allá del precio transado en las distintas bolsas de metales, el valor presente neto del producto puede haber sido infravalorado, dado la falta de consideración en las valoraciones pasadas de la excepcionalidad del producto no renovable. Otra razón para reestimar el precio del cobre más allá de las de los deflactores por tasas de cambio de las monedas, es lo relativo del precio de mercado/exportación cuando se considera en relación a los productos importados. Diversos trabajos han comenzado a abordar una reestimación de los términos de intercambio para los países 
Latinoamericanos, en una línea de investigación necesaria para testear la hipótesis Prebisch -Singer (Ocampo and Parra-Lancourt, 2010; Erten and Ocampo, 2013; Kuntz-Ficker, 2017). Por último, una consideración no menor cuando se analizan los precios reales, es la ausencia de un análisis de los costos que tienen los distintos commodities. Aunque el precio "real" esté deflactado, el precio en cuestión sigue siendo una referencia de la transacción del commodity en el mercado y no cuánto cuesta producirlo ${ }^{4}$. En el caso del cobre, los costos se han incrementado notablemente, dado la extenuación de las vetas más ricas, en términos de la ley del mineral, y la consiguiente inversión en capital fijo requerida para extraer material con menor contenido. También, los costos labores han tenido un incremento apreciable, dada la dificultad para encontrar personal capacitado en los últimos ańos y las compensaciones salariales proporcionales a los traslados a zonas remotas.

En la gráfica 1, podemos apreciar el valor del cobre en números índices, a través de diversas series de precios que cubren más de 200 años de historia.

Figura 1. Índices de precios del cobre. Diversas ESTIMACIONES $1820-2021^{5}$

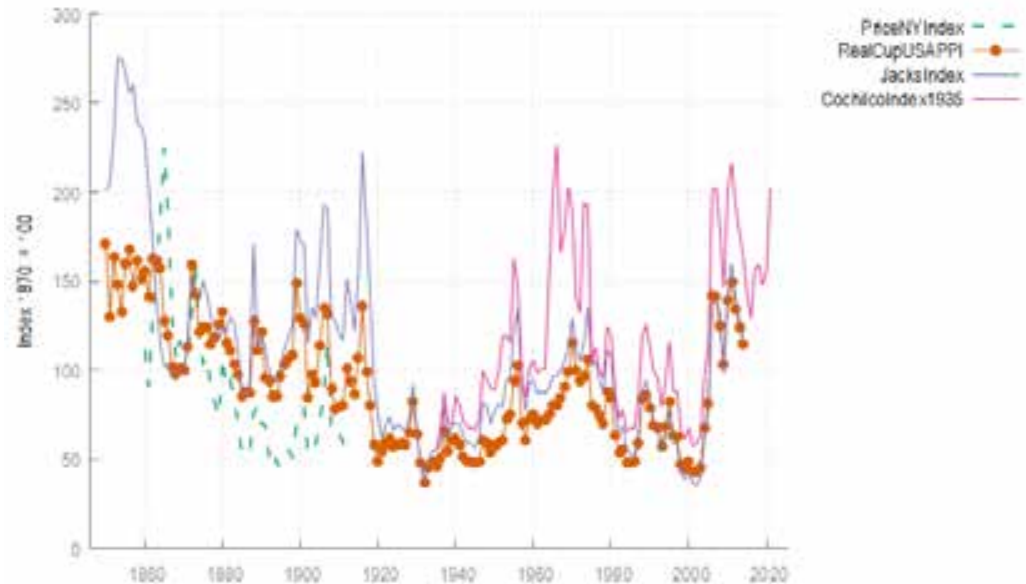

Fuentes: (Jacks, 2018; Stuermer, 2018) and Cochilco, acceso el 24 de mayo de 2021. 
En esta gráfica ya podemos apreciar las diferencias en cuanto a afirmaciones del tipo "mayor precio histórico". La serie de datos más larga que contamos con respecto al producto es la elaborada por (Schmitz, 1979), pero esta no cubre los últimos 45 ańos. Las series elaboradas por (Harvey et al., 2010; Jacks, 2018; Stuermer, 2018) tienen distintos periodos, pero todas se componen de los mismas fuentes (en el caso del cobre). Estas fuentes son la bolsa de metales de Londres, los anuarios mineros norteamericanos y la bolsa de futuros de Nueva York (COMEX). La particularidad de estas fuentes hace que las series tengan tendencias similares, pero haya diferencias relevantes cuando se trata de los niveles. Entre las diversas explicaciones, una es que el precio "internacional" no refleje los precios que se estén pagando o cotizando en los países exportadores, una particularidad que fue analizada para el caso noruego y chileno en (Ducoing, Badia-Miró and Anna, 2021). No obstante, con las series presentadas en la figura 1, ya es claro que afirmaciones sobre la historicidad y excepcionalidad del precio actual deberían ser tomadas con cautela. El precio deflactado actual es importante en términos monetarios y se compara en números índices con los ciclos excepcionales de la década del 1960, el largo ciclo relacionado al auge de la electrificación de principios del siglo pasado y el reciente "súper ciclo" de las materias primas, pero estos ciclos fueron continuados en el tiempo y en periodos donde los costos de extracción del cobre eran menores. Aunque a primera vista el precio del cobre pareciera extraordinariamente elevado en una perspectiva de corto plazo (últimos cinco ańos), esta afirmación no es del todo correcta teniendo en cuenta una mirada de largo plazo.

Para finalizar esta corta reflexión sobre la valorización de los recursos naturales, y específicamente el cobre, existen diversas líneas de investigación dentro de la historia económica que deberían ser profundizadas y que merecen mayor atención de la que han recibido hasta el momento. En primer lugar, las fuentes del precio del cobre en el largo plazo, que podría mejorar los futuros pronósticos y ayudar a los países productores a planificar mejores políticas fiscales (royalties) para aprovechar estos ciclos. Para desarrollar esta línea de 
investigación, el primer elemento a considerar sería un análisis de series temporales similar al desarrollado por (Jacks, 2018) para identificar los quiebres estructurales y los ciclos del precio del cobre. En segundo lugar, en los periodos identificados, un análisis de las fuentes de la demanda del producto tendría que recibir un tratamiento especial, como son los aumentos de la capacidad instalada de electricidad, la producción de barcos que requerían revestimiento y los potenciales productos reemplazantes/complementarios del mineral en cuestión. Un mejor conocimiento de las fluctuaciones de los precios de los recursos naturales debería aportar ideas y estrategias para afrontar la demanda esperada en los próximos ańos debidos a los imperiosos esfuerzos en pos de economías más sustentables. El rol de la historia económica se hace fundamental, puesto que las perspectivas de largo plazo han sido poco consideradas en estos debates.

\section{REFERENCIAS}

BLUM, M., DUCOING, C. AND MCLAUGHLIN, E. (2017) 'A Sustainable Century? Genuine Savings in Developing and Developed Countries, 1900 - 2000', in Hamilton, K. and Hepburn, C. (eds) National Wealth What Is Missing, Why It Matters. Oxford Univ Pr.

DUCOING, C. et al. (2018) 'Natural Resources Curse in the Long Run? Bolivia, Chile and Peru in the Nordic Countries' Mirror', Sustainability 2018, Vol. 10, Page 965. Multidisciplinary Digital Publishing Institute, 10(4), p. 965. doi: 10.3390/ SU10040965.

DUCOING, C., BADIA-MIRÓ, M. AND ANNA, C. (2021) 'Copper Prices and Heterogeneity: The Chilean and Norwegian Cases, 1850-1913', in Natural Resources and Divergence: A Comparison of Andean and Nordic Trajectories. Palgrave Macmillan.

ERTEN, B. AND OCAMPO, J. A. (2013) 'Super Cycles of Commodity Prices Since the Mid-Nineteenth Century', World Development, 44, pp. 14-30. doi: 10.1016/j. worlddev.2012.11.013. 
HARTWICK, J. (1977) 'Intergenerational equity and the investing of rents from exhaustible resources', The american economic review. Available at: http://www.jstor.org/stable/1828079 (Accessed: 25 May 2016).

HARVEY, D. I. et al. (2010) 'The Prebisch-Singer Hypothsis: Four Centuries of Evidence', Review of Economics and Statistics, 92(2), pp. 367-377. doi: 10.1162/rest.2010.12184.

JACKS, D. S. (2018) 'From boom to bust: a typology of real commodity prices in the long run', Cliometrica. Springer Berlin Heidelberg, pp. 1-20. doi: 10.1007/s11698-018-0173-5.

KUNTZ-FICKER, S. (2017) 'Latin America's First Export Era: Reassessing Its Economic Contribution', in The First Export Era Revisited. Cham: Springer International Publishing, pp. 1-35. doi: 10.1007/978-3-319-62340-5_1.

OCAMPO, J. A. AND PARRA-LANCOURT, M. (2010) 'The terms of trade for commodities since the mid-19th century', Revista de Historia Económica / Journal of Iberian and Latin American Economic History. Cambridge University Press, 28(01), p. 11. doi: 10.1017/S0212610909990085.

SCHMITZ, C. J. (1979) World Non-ferrous Metal Production and Prices, 1700-1976. Cass. Available at: https://books.google. com/books?id=up6DQgAACAAJ\&pgis=1 (Accessed: 28 October 2015).

STUERMER, M. (2018) '150 YEARS of BOOM and BUST: WHAT DRIVES MINERAL COMMODITY PRICES?', Macroeconomic Dynamics. Cambridge University Press, 22(3), pp. 702-717. doi: 10.1017/S136510051600050X.

WRIGHT, G. (2015) 'The USA as a Case Study in Resource-Based Development', in Badia-Miró, M., Pinilla, V., and Willebald, H. (eds) Natural resources and economic growth: learning from history. Routledge. 
Notas de Página

https://www.emol.com/noticias

Economia/2021/05/07/1020174/cobre-supea-precio-historico.html acceso el 08 de mayo de 2021

${ }^{2}$ Cochilco publica diariamente una actualización de estos precios, junto con los promedios mensuales y anuales. Dos son las principales fuentes de las cuales Cochilco basa sus estimaciones. Por una lado, la Bolsa de metales de Londres (London Metal Exchange en el original en Inglés https://www.lme.com/) y le mercado de futuros de Nueva York (New York Mercantile Exchange and Commodity Exchange https://www.cmegroup.com/)

${ }^{3}$ Adjusted Net Savings o Genuine Savings en el original en inglés. Los ahorros genuinos se definen como el monto del producto no consumido en una economía en un periodo determinado. Este ahorro se ajusta descontado la depreciación del capital físico, las rentas en recursos natural, las emisiones de CO2 y sumando las inversiones en capital humano. Para una descripción más detallada, (Blum, Ducoing and McLaughlin, 2017).

${ }^{4}$ Según algunos reportes hecho por Cochilco, se ha estimado un aumento del costo medio de operación desde los $90 \mathrm{cUS} \$ / \mathrm{lb}$ del año 2005 hasta los 217 cUS\$/lb el año 2014, algo cercano a un $10 \%$ anual. https://www.cochilco.cl/Listado\%20Temtico/Informe caracterizacion de los costos.pdf Acceso 24 de Mayo de 2021.

5 Para el año 2021 se tomó en cuenta el promedio de los meses de enero hasta abril.

Fecha recepción: 7 de junio de 2021

Fecha aceptación: 15 de junio de 2021

Fecha versión final: 17 de junio de 2021 